Aims. To compare the Emergency Department (ED) referrals to psychiatry in a suburban versus an urban setting over a onemonth to evaluate mental health presentations characteristics across two locations.

Method. This study was a retrospective cross-sectional study examining ED referrals to psychiatry in an inner-city and suburban centre over one month; - one based in an inner-city setting, the other based in a suburban area outside the city. The anonymised data were collected from both hospital's electronic patient records and analysed. The authors collected data on gender, age, employment, housing, the clinical problem at presentation, time of assessment and admissions. Descriptive data and hypothesis testing were performed where appropriate using Statistical Package for Social Sciences SPSS ${ }^{\circledR}$ version 26.

Result. The total number referred was 213: inner-city $n=109$ and suburban $\mathrm{n}=104$. The inner-city saw a younger population; $47 /$ 109 (43\%) were aged between 20 and 29 years, compared with 28/104 (27\%) of suburban presenters (P-value 0.0134). A higher number of presenters were aged over 60 years in the suburban centre $n=13 / 104(12.5 \%)$ versus the inner-city centre $3 / 109$ $(2.8 \%)$ (P-value 0.0084$)$. In the inner-city, the proportion of homeless presenters was significantly higher at 30/109 (28\%) versus $5 / 104(4.8 \%)$ in the suburban setting $(\mathrm{P}<0.0001)$. Presentations related to substances were highest, a total of 73 (34.3\%) across both centres, with no significant difference in clinical presentations across the two centres. The majority were seen in the on-call period, 74/109 (67.9\%) in the inner-city centre and $66 / 104(63.5 \%)$ in the suburban centre. The psychiatric admission rate was significantly different between the two centres, with 33/ $109(30.3 \%)$ patients admitted to the inner-city centre and 13/ $104(12.5 \%)$ patients admitted to the suburban centre (P-value $0.002)$.

Conclusion. A large proportion of ED referrals to psychiatry constitute patients with unmet social and addiction needs. The variance in capabilities of liaison psychiatry (LP) and ED services means the local population's needs may not always be adequately catered for within a typical LP setting, which in the Irish context is predominantly driven by medical and nursing staff. This study highlights many patients attend the ED who may be better assessed directly by the community as per our National Emergency Program policies. This prompts consideration of expanding both ED and community services to comprise a more integrated, multidisciplinary-resourced, 24/7 care model.

\section{Audit of high dose antipsychotics (HDA) prescribing on high dependency unit (HDU) and acute ward, Nepean Mental Health Centre, Nepean Blue Mountains Local Health District, Sydney, Australia \\ Adil Jawad ${ }^{*}$ Kristof Mikes-Liu, Christopher Mah and Khyati Roy Nepean Blue Mountains Local Health District ${ }^{*}$ Corresponding author.}

doi: 10.1192/bjo.2021.862

Aims. To measure the rate of patients receiving high dosage antipsychotics.

To review the adherence to maximum recommended doses of antipsychotics as per the product information approved by Australian Therapeutic Goods Administration, product information approved by Medsafe (the New Zealand Medicines and Medical Devices Safety Authority) and Therapeutic Guidelines (Psychotropic Writing Group, 2013)
Background. High dose antipsychotics or combination of antipsychotics are associated with significant adverse effects including QTc prolongation, arrhythmias, sudden cardiac death, seizures, increased incidence and severity of adverse effects, longer hospital stay and possibly increased mortality. High dose antipsychotic prescribing may arise as a result of EITHER single antipsychotic drug prescribed at a daily dose above the recommended limit (High Dose single drug) OR More than one antipsychotic prescribed concurrently where the sum of doses given expressed as a percentage of the SPC maximum of each drug exceeds 100\% (High-Dose through the prescribing of multiple drugs).

Method. The data were gathered from all the drug charts for all patients admitted to HDU and Acute ward on 9th April 2019. The Audit standards were 1) Individual antipsychotic dose should be within recommended limit as $100 \%$ and 2) Combined antipsychotics should be within recommended limit as $100 \%$

Result. Total number of patients on both the HDU and Acute wards $=33$

Total number of patients on antipsychotics $=30$

Number of patients on $>100 \%$ of recommended cumulative dosage $=13 / 30=43.3 \%$

Number of patients on $>100 \%$ maximum limits of regular antipsychotics $=3=10 \%$

Number of patients on $>100 \%$ maximum limits of PRN antipsychotics $=0 / 30$

Number of patients on 2 antipsychotic $=18 / 30=60 \%$

Number of patients on 3 antipsychotic $=8 / 30=26.6 \%$

Number of patients on 4 antipsychotic $=2 / 30=6.6 \%$

Conclusion. Out of the 30 patients on antipsychotics, almost half were on more than $100 \%$ of the recommended cumulative maximum limits of antipsychotics doses, almost $2 / 3$ rds were on 2 or more antipsychotic and a quarter on 3 or more. This can be associated with significant adverse effects including QTc prolongation, arrhythmias, sudden cardiac death, seizures, increased incidence and severity of adverse effects, longer hospital stay and possibly increased mortality. There is a need to review PRN antipsychotics prescribed as a norm, clear documentation and need for a protocol for increased vital sign monitoring for patients on high dose antipsychotic treatment.

\section{Audit report of physical health examination and} baseline investigations on high dependency unit (HDU) and acute ward, Nepean Mental Health Centre, Sydney, Australia

Adil Jawad*, Kristof Mikes-Liu, Christopher Mah, Amgad Elmakki and Riffat Fatima

Nepean Hospital, Mental Health Centre

${ }^{\star}$ Corresponding author.

doi: 10.1192/bjo.2021.863

Aims. All patients on High Dependency Unit (HDU) and Acute Ward, Mental Health Centre, Nepean Hospital, were included in a cross-sectional audit on 22nd January 2020. Out of a total of 43 patients admitted on both these wards, $88.4 \%$ had baseline blood tests done, but almost half did not have baseline ECG done and $1 / 3$ rd did not have a physical examination done. The physical examination on admission on these wards is better than in 2017 \& 2018 when half and more than $1 / 3$ rd respectively did not have physical examination done. 